\title{
The Atmospheric Imaging Assembly (AIA) on the Solar Dynamics Observatory (SDO)
}

\author{
James R. Lemen • Alan M. Title • David J. Akin • Paul F. Boerner • Catherine Chou • \\ Jerry F. Drake • Dexter W. Duncan • Christopher G. Edwards • \\ Frank M. Friedlaender • Gary F. Heyman • Neal E. Hurlburt • Noah L. Katz • \\ Gary D. Kushner • Michael Levay • Russell W. Lindgren • Dnyanesh P. Mathur • \\ Edward L. McFeaters • Sarah Mitchell • Roger A. Rehse • Carolus J. Schrijver • \\ Larry A. Springer · Robert A. Stern - Theodore D. Tarbell • Jean-Pierre Wuelser • \\ C. Jacob Wolfson - Carl Yanari · Jay A. Bookbinder • Peter N. Cheimets • \\ David Caldwell • Edward E. Deluca • Richard Gates · Leon Golub • Sang Park • \\ William A. Podgorski • Rock I. Bush • Philip H. Scherrer • Mark A. Gummin • \\ Peter Smith • Gary Auker • Paul Jerram • Peter Pool • Regina Soufli • David L. Windt • \\ Sarah Beardsley • Matthew Clapp · James Lang • Nicholas Waltham
}

Received: 6 December 2010 / Accepted: 11 April 2011 / Published online: 2 June 2011

(C) Springer Science+Business Media B.V. 2011

\begin{abstract}
The Atmospheric Imaging Assembly (AIA) provides multiple simultaneous highresolution full-disk images of the corona and transition region up to $0.5 R_{\odot}$ above the solar limb with 1.5-arcsec spatial resolution and 12-second temporal resolution. The AIA consists of four telescopes that employ normal-incidence, multilayer-coated optics to provide
\end{abstract}

The Solar Dynamics Observatory

Guest Editors: W. Dean Pesnell, Phillip C. Chamberlin, and Barbara J. Thompson

J.R. Lemen $(\varangle)$ · A.M. Title · D.J. Akin · P.F. Boerner · C. Chou · J.F. Drake · D.W. Duncan ·

C.G. Edwards · F.M. Friedlaender · G.F. Heyman · N.E. Hurlburt · N.L. Katz · G.D. Kushner ·

M. Levay - R.W. Lindgren · D.P. Mathur · E.L. McFeaters · S. Mitchell · R.A. Rehse · C.J. Schrijver ·

L.A. Springer · R.A. Stern · T.D. Tarbell · J.-P. Wuelser · C.J. Wolfson · C. Yanari

Solar and Astrophysics Laboratory, Lockheed Martin Advanced Technology Center, Bldg. 252, Org.

ADBS, 3251 Hanover St., Palo Alto, CA 94304, USA

e-mail: lemen@Imsal.com

Present address:

L.A. Springer

Department of Physics, Montana State University-Bozeman, P.O. Box 173840, Bozeman, MT 59717, USA

J.A. Bookbinder · P.N. Cheimets · D. Caldwell · E.E. Deluca · R. Gates · L. Golub · S. Park ·

W.A. Podgorski

Smithsonian Astrophysical Observatory, 60 Garden Street, Cambridge, MA 02138, USA

R.I. Bush · P.H. Scherrer

W. W. Hansen Experimental Physics Laboratory, Center for Space Science and Astrophysics, Stanford University, Stanford, CA 94305, USA

M.A. Gummin

Alias Aerospace, Inc., 1731 Saint Andrews Court, St. Helena, CA 94574, USA 
narrow-band imaging of seven extreme ultraviolet (EUV) band passes centered on specific lines: Fe XVIII (94 ̊), Fe VIII, XXI (131 ̊), Fe IX (171 ̊), Fe XII, XXIV (193 ̊), Fe XIV (211 $\AA$ ), He II (304 $\AA$ ), and Fe XVI (335 ̊). One telescope observes C IV (near $1600 \AA$ ) and the nearby continuum $(1700 \AA)$ and has a filter that observes in the visible to enable coalignment with images from other telescopes. The temperature diagnostics of the EUV emissions cover the range from $6 \times 10^{4} \mathrm{~K}$ to $2 \times 10^{7} \mathrm{~K}$. The AIA was launched as a part of NASA's Solar Dynamics Observatory (SDO) mission on 11 February 2010. AIA will advance our understanding of the mechanisms of solar variability and of how the Sun's energy is stored and released into the heliosphere and geospace.

Keywords Solar corona $\cdot$ Solar instrumentation $\cdot$ Solar imaging $\cdot$ Extreme ultraviolet

\section{Introduction}

The primary goal of the Solar Dynamics Observatory is to understand the physics of solar variations that influence life and society. It achieves that goal by targeted basic research focused on determining how and why the Sun varies, and on improving our understanding of how the Sun drives global change and space weather. As one of the SDO instruments designed to meet this goal, the Atmospheric Imaging Assembly (AIA) focuses on the evolution of the magnetic environment in the Sun's atmosphere, and its interaction with embedded and surrounding plasma. Figure 1 shows the four AIA telescopes mounted to the SDO spacecraft, and Figure 2 shows schematically the layout of the telescopes with respect to their wavelength band passes.

The images of the corona taken by Yohkoh, the Extreme ultraviolet Imaging Telescope (EIT) on the Solar and Heliospheric Observatory (SOHO), the Sun Earth Connection Coronal and Heliospheric Investigation (SECCHI) on the Solar Terrestrial Relations Observatory (STEREO), and the Transition Region and Coronal Explorer (TRACE) have shown that all coronal structures evolve in density, temperature, and position on time scales as short as seconds. Conditions within coronal-loop volumes and open magnetic structures appear to depend primarily on "local" conditions, i.e. on conditions determined by the path of the field line, or loop, from end to end in the photosphere. Combined with the marked temporal evolution of the atmospheres contained within coronal loops or open-field regions on relatively short time scales, EUV images are dissimilar for different band passes. Apart from

\footnotetext{
P. Smith

Harvard University-Smithsonian Astrophysical Observatory, 60 Garden Street, Cambridge, MA 02138, USA
}

G. Auker · P. Jerram · P. Pool

e2v technologies, 106 Waterhouse Lane, Chelmsford, Essex CM1 2QU, UK

R. Soufli

Lawrence Livermore National Laboratory, Livermore, CA 94550, USA

D.L. Windt

Reflective X-ray Optics LLC, New York, NY 10027, USA

S. Beardsley $\cdot$ M. Clapp $\cdot$ J. Lang $\cdot$ N. Waltham

Rutherford Appleton Laboratory, Harwell Business Innovation Campus, Didcot, Oxon, OX11 0QX, UK 
Figure 1 The AIA telescopes with their guide telescopes as seen mounted on the SDO spacecraft's instrument module during integration in the clean room at NASA's Goddard Space Flight Center.
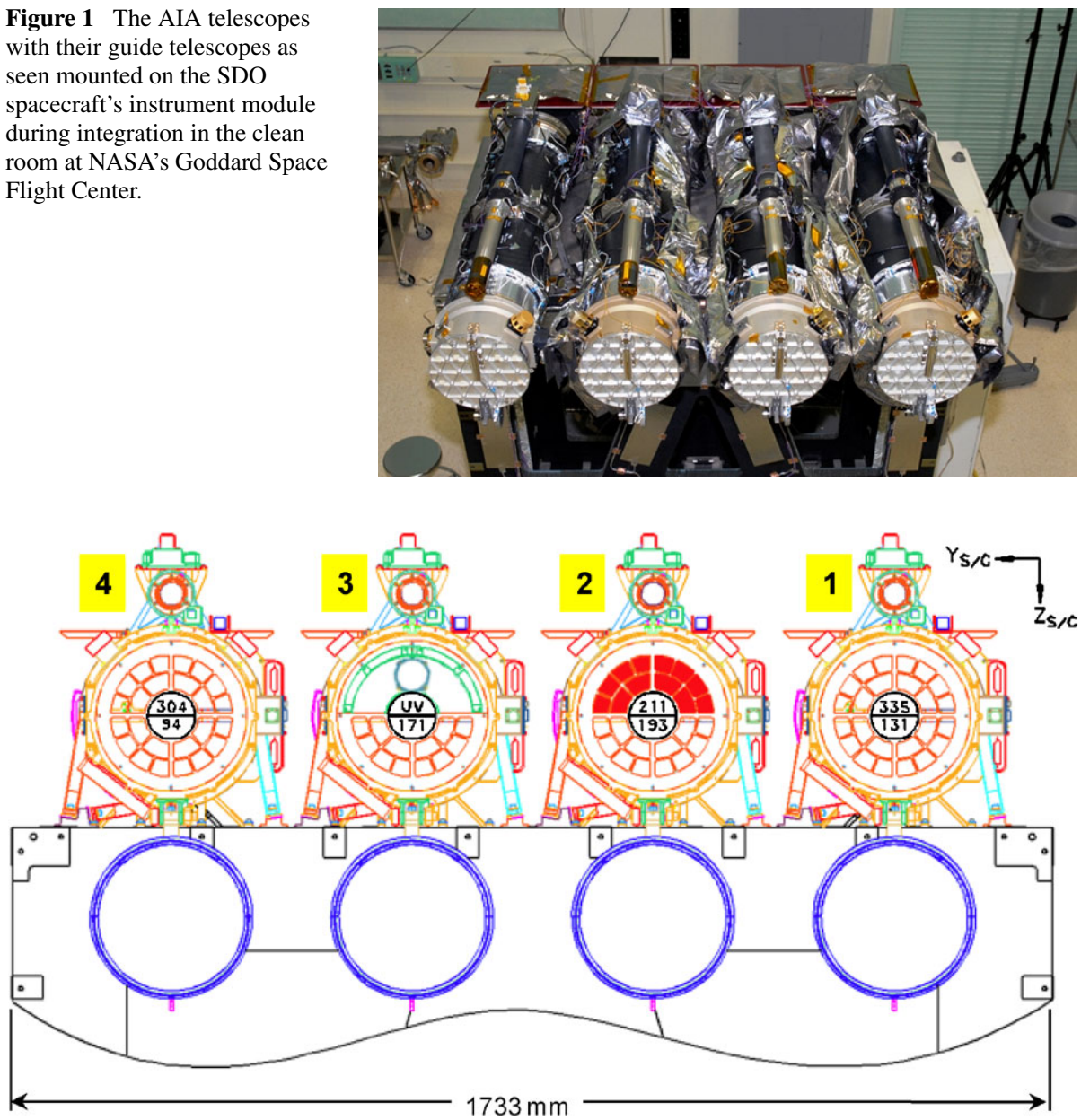

Figure 2 The layout of the wavelength channels or band passes in each of the four AIA telescopes. Telescope 2 has an aperture blade to select between wavelength channels. The other telescopes rely on filters in filter wheels to select between channels. The top half of telescope number 3 has a $\mathrm{MgF}_{2}$ window with a coating centered at $1600 \AA$.

thermal evolution, there is an abundance of waves, flows, and impulsive phenomena that occur on short time scales of minutes or less.

The coronal magnetic field itself evolves on time scales that range from seconds to years. Slowest is the evolution of the largest-scale field. On shorter time scales, electrical currents that are induced in, and carried with, the magnetic field may build up over weeks or months, while release of those stresses may take only a fraction of a minute. Yohkoh and SOHO have shown, moreover, that there are no purely local field topologies: the short- and long-term evolution of the corona is affected by both nearby and distant magnetic sources.

AIA was designed to study these characteristics of the Sun's dynamic magnetic field and the coronal response to it. AIA provides the following essential capabilities:

i) a view of the entire corona at the best feasible resolutions compatible with SDO's constraints, providing coverage of the full thermal range of the corona; 
ii) a high signal-to-noise ratio for two- to three-second exposures that reaches 100 in quiescent conditions for the low-temperature coronal-imaging channels and during flaring in the higher-temperature channels, with a dynamic range of up to 10000 ; and

iii) essentially uninterrupted viewing for months at a time at a temporal resolution of approximately 10 to 12 seconds, and sometimes faster to study energetic transient phenomena.

These capabilities are met by a design that includes the following.

i) Four 20-cm, dual-channel, normal-incidence telescopes, which observe a 41 arcmin field of view in ten EUV and UV channels, with 0.6-arcsec pixels and $4096 \times 4096$ CCDs. Four of the EUV wavelength bands open new perspectives on the solar corona, having never been imaged or imaged only during brief rocket flights. The set of six EUV channels that observe ionized iron allow the construction of relatively narrow-band temperature maps of the solar corona from below $1 \mathrm{MK}$ to above $20 \mathrm{MK}$.

ii) Detectors with a full well of at least 150000 electrons, with typically $18 \mathrm{e}^{-}$photon $^{-1}$ (for $193 \AA$ ), and a readout noise of $<25 \mathrm{e}^{-}$, and data compression that is nearly lossless.

iii) A standard baseline observing program running most of the time, while observing continuously from SDO's geo-synchronous orbit. AIA has the capability to adjust its observing program to changing solar conditions in order to implement observing programs that are optimized to meet the requirements of specific scientific objectives. This allows, for example, a two-second cadence in a reduced field of view using four wavelengths for flare studies.

With these capabilities, AIA enables us to observe the changing topology of the magnetic field even as the coronal plasma is changing in temperature. In its standard operating mode, the AIA images the entire Sun ten times faster per wavelength than TRACE at twice the number of coronal channels, with a 16-fold increase in the number of pixels per image. Compared with the full-disk SOHO/EIT (Delaboudinière et al., 1995), AIA runs at $700 \times$ the usual EIT image frequency, with 16 times more pixels per image, in typically eight instead of four channels. Compared to EIT and TRACE, the AIA thus represents an increase in the information rate for coronal observations by a factor of 400 to 22000 . AIA also advances on the Hinode/X-Ray Telescope (XRT) (Golub et al., 2007) and SECCHI/Extreme Ultra-Violet Imager (EUVI) (Howard et al., 2008) telescope designs, each which contained $2 \mathrm{k} \times 2 \mathrm{k}$ pixel CCDs with one and 1.6-arcsec pixel sizes, respectively. And SDO's Helioseismic and Magnetic Imager (HMI) provides, among other things, high spatial resolution line-of-sight magnetograms at a cadence suitable for the evolution of the photosphere that is an order of magnitude faster than SOHO/MDI (Schou et al., 2011).

The basic AIA observables are full-Sun intensities at a range of wavelengths. In combination with the higher-level and metadata products these comprise the data archive which is freely accessible to the research community; the archive and its interfaces (including those for data requests and archive queries) are described by Hurlburt et al. (2011).

\section{Science Overview}

The AIA investigation covers a broad range of science objectives that focus on five core research themes that both advance solar and heliospheric physics in general and provide advanced warning of coronal and inner-heliospheric disturbances of interest to the Living With a Star program, i.e., global change, space weather, human exploration of space, and technological infrastructure in space and on Earth. 
i) Energy input, storage, and release: the 3D dynamic coronal structure, including reconnection and the effects of coronal currents.

ii) Coronal heating and irradiance: origins of the thermal structure and coronal emission, to understand the basic properties of the solar coronal plasma and field, and the spatiallyresolved input to solar spectral irradiance, as observed by, e.g., SDO's Extreme ultraviolet Variability Explorer (EVE).

iii) Transients: sources of radiation and energetic particles.

iv) Connections to geospace: material and magnetic field output of the Sun.

v) Coronal seismology: a diagnostic to access sub-resolution coronal physics.

These five themes guide the AIA science investigation, and their fundamental observational needs have shaped the design of the instrument.

\subsection{Energy Input, Storage, and Release: The Dynamic Coronal Structure}

All coronal activity relies on energy dissipation within the plasma contained in the Sun's magnetic field. One of the primary objectives of the NASA's Living with a Star program, and of the AIA science investigation in particular, is to understand how energy is brought into the coronal field, how it is stored there, and how it is released. One key part of that objective is to understand the geometry of the magnetic field, and its evolution subject to external forcing and internal energy dissipation.

Plasma motions in and below the solar photosphere force the embedded magnetic field to move with the flows. These motions apply stress to the outer-atmospheric field on a broad range of spatial and temporal scales. This stress propagates throughout the outer atmosphere in the form of a variety of wave types and frequencies, as well as a broad spectrum of slower changes associated with evolving induced currents. Key questions to be answered are: How are these perturbations imposed upon the corona? How do they propagate? How do the electric currents close either within the atmosphere or through the photosphere?

TRACE observations reveal that the stresses that are applied to the coronal field by the photospheric motions lead to a magnetic field that is only moderately tangled on scales of the granular motions up to the largest scales, despite theoretical expectations that fieldline braids should persist for long times in a coronal environment where reconnection was expected to proceed only slowly. Instead of a highly tangled field, however, TRACE images show that small-scale braids, driven by convective motions from the arcsecond scale of the granulation upward, are only seen around filaments and prominences. Even then, the images suggest that loop twists rarely exceed about half a turn. This implies rapid dissipation of the induced currents, which is mimicked by some recent numerical simulations. The fidelity of these simulations does not approach the real corona, however, with anticipated (but maybe not realized) magnetic Reynolds numbers for the bulk of the coronal volume many orders of magnitude larger than in the simulated volume. In contrast to the apparently efficient smoothing out of the small-scale complexity, many filament configurations erupt, requiring substantial amounts of stored energy. Why do small-scale twists dissipate readily, whereas large-scale stresses persist for long times in filament and sigmoid-field configurations which can eventually lead to instabilities?

\subsection{Coronal Heating and Irradiance: Thermal Structure and Emission}

Solar radiation at UV, EUV, and SXR wavelengths plays a significant role in the determining the physical properties of the Earth's upper atmosphere. Variations in solar radiation at these wavelengths drive changes in the density and ionization of the Earth's thermosphere 
and ionosphere, impacting the performance of ground-based communications systems and spacecraft in low-Earth orbit. Long-term observations of the solar irradiance with the required accuracy have proven difficult, however, and much of our knowledge of the solar irradiance and its variability remains uncertain.

Observations over the past several decades have clearly established that solar variability at UV, EUV, and SXR wavelengths is tied to the variability of the Sun's surface magnetic fields. Past studies of the Sun and Sun-like stars has revealed that the primary determinant of the radiation leaving the corona is the total magnetic flux threading the photosphere. At present, however, we do not possess a detailed understanding of how magnetic energy is released within the solar corona. Thus we cannot use our knowledge of the Sun's magnetic fields to model and predict changes in the solar irradiance. Such calculations are an invaluable aid to interpreting and extending direct measurements of the solar irradiance. One of the primary objectives of the SDO mission is to develop a physical understanding of solar irradiance and its variability, specifically at (E)UV wavelengths, with close collaboration between the science teams of AIA and of SDO's Extreme ultraviolet Variability Experiment (EVE: Woods et al., 2011). AIA images will show the locations and structural/thermal changes associated with EUV irradiance changes measured with high precision by EVE.

\subsection{Transients: Sources of Radiation and Energetic Particles}

Most of the time, the coronal field appears to evolve smoothly. At times, however, massive explosions or eruptions disturb the coronal field. Many are not strong enough to rupture the encapsulating coronal magnetic field, and only the energetic radiation escapes toward Earth. Some, in contrast, are associated with opening magnetic fields into the heliosphere; these coronal eruptions appear to be the counterparts of coronal mass ejections. What triggers the eruptions or explosions? What determines whether the field confines the eruption to the corona or allows coupling into the heliosphere?

Flares, CMEs, filament destabilizations, sprays, and other transients are sources of radiation and energetic particles, and therefore prime drivers of violent solar weather. Transients result from the sudden conversion of magnetic energy into bulk, thermal, and non-thermal energy as the magnetic field reconnects. Theories of 3D reconnection are still in early stages of development, and advances in its understanding are hampered by observational limitations.

AIA is designed to make the next leap forward in understanding transient initiation and evolution. Its high-cadence, full-disk, multi-temperature observations are revealing the reorganizing field in the initial phases of flares and of filament eruptions, as well as the later evolution as the field relaxes into its new state. Particularly promising is the inclusion of four EUV pass bands that observe the evolution of the coronal plasma for the first time at near-arcsecond resolution for temperatures between 3 to $20 \mathrm{MK}(94,131,211$, and $335 \AA$ ).

\subsection{Connections to Geospace: Material and Magnetic-Field Output of the Sun}

The solar wind, the embedded magnetic field, and eruptive perturbations in the form of CMEs drive the variations in the space surrounding the Earth and other planets. The dynamic connections between Sun and geospace are a cornerstone of the ILWS program. To understand how the Sun's variability affects life and society, we must understand how the products of this variability are transported into and through the heliosphere, and how they interact with the Earth's magnetic field and atmosphere. The AIA investigation is expected to make substantial quantitative advances in many areas relevant to this problem. At the foundation of this expectation lies the improved understanding of the global coronal field and its 
extension into the heliosphere. In Sections 2.2 and 2.3 we discussed this for the pathways for escape of energetic particles into the heliosphere, the irradiance that affects the ionosphere and below, and the triggering of flares and CMEs. Here we focus on the magnetized solar wind and its perturbations.

The solar wind flows out radially into the heliosphere dragging along the magnetic-field lines that are forced "open" within the first few radii from the Sun. The successes of simple concepts such as the potential-field source-surface (PFSS) model suggest that more realistic and detailed models should explain why this is. What determines which field lines will open? Why does the PFSS work so well? The latter is particularly interesting because the PFSS model does not incorporate field dynamics. How is field opened, and how is it closed again as the connections evolve? This is somehow connected to the properties of the solar wind, which we know depend on the field geometry and strength. But although that dependence is empirically constrained, we still need to learn what determines the physical properties of the solar wind.

The eruptive coronal mass ejections perturb the background solar wind. How do they evolve through the coronal field? How do they couple into, and propagate through, the heliosphere on their way to the Earth and the other planets? HMI and AIA data will enable modelers to addresses these questions by advancing beyond PFSS models to full MHD models of the coronal and inner heliosphere.

\subsection{Coronal Seismology: A Diagnostic to Study Coronal Waves and Oscillations}

Recent observations from SOHO and TRACE show a variety of oscillation modes in the transition region and corona. These observations opened up the promising new field of coronal seismology. By studying the properties, excitation, propagation and decay of these oscillations and waves, we can reveal fundamental physical properties of the solar transition region and corona, such as the magnetic field, density, temperature, and viscosity.

Examples of seismic responses of the corona have been found in large-scale coronal Moreton and EIT waves, in polar plumes, sunspot fields, upper transition-region moss, and what appear to be ordinary loops. Perhaps most striking are the transverse oscillations seen by TRACE. Longitudinal oscillations and waves have also been observed in the upper transition-region moss and in coronal loops, both in cooler loops with TRACE and in hot $\left(10^{7} \mathrm{~K}\right)$ loops with the Solar Ultraviolet Measurements of Emitted Radiation (SUMER) spectrometer.

There are still many unresolved issues about these waves and oscillations. How do they get excited? Why does only a fraction of the observed flares lead to clear oscillations? Are longitudinal waves in coronal loops with five-minute periods related to $p$ modes? How do any of these waves propagate? Which of the many possible theoretical mechanisms can explain the unexpectedly rapid decay of some of these waves? These are some of the questions that we need to answer before we can seismically probe coronal physics.

The AIA design enables us to develop the necessary improved understanding of the properties and other unresolved issues of the observed waves. The high cadence of AIA will extend the parameter space to higher frequencies (by a factor of two-four compared with TRACE). The broad, simultaneous temperature coverage positions us to study waves in parts of active regions that have not yet been seen. Guided by significant advances in the theory of these waves (from 3D MHD simulations), and complemented with spectroscopic measurements of densities and line-of-sight velocities (e.g. by the EUV Imaging Spectrometer on Hinode), and reliable magnetic-field extrapolations (e.g. from HMI and AIA), AIA will fully exploit the potential of coronal seismology. 

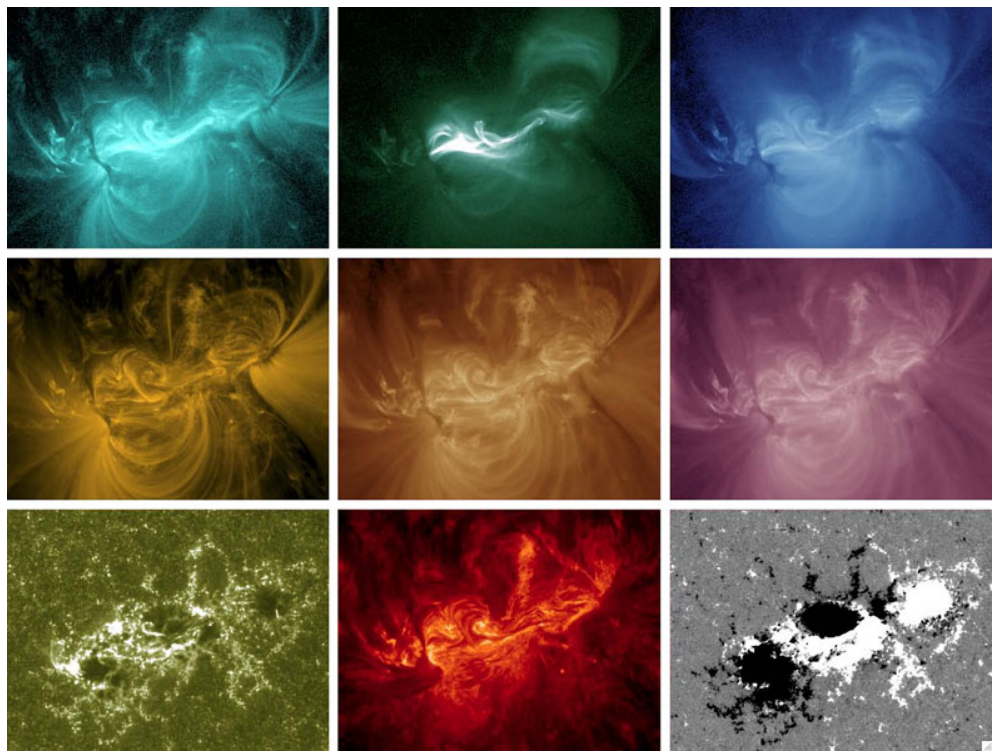

Figure 3 Images of an active region observed on 15 February 2011 at 01:45 UT with AIA. The top row panels are observations from the 131-, 94-, and 335- $\AA$ channels (from left to right). The middle row panels are from the 171-, 193-, and 211- $\AA$ channels, and the bottom row panels are from 1600 and $304 \AA$ (left and middle, respectively). The bottom right corner panel is an HMI line-of-sight magnetogram showing the same field of view. The AIA images have been processed to Level 1.5 and each contains $480 \times 360$ pixels, which corresponds to $288 \times 216$ arcsecs.

\section{Instrument Overview}

The AIA instrument consists of four generalized Cassegrain telescopes that are optimized to observed narrow band passes in the EUV in order to observe solar emissions from the transition region and corona. An active region observed on 15 February 2011 is shown in Figure 3 for the seven EUV channels, the 1600- $\AA$ channel, and an HMI line-of-sight magnetogram. This mosaic of images illustrates how the various instrument-response functions sample the temperature-dependent structure of the solar corona. Table 1 lists the primary ions for each band pass and their characteristic emission temperatures, along with the types of solar features that may be observed.

Each AIA f/20 telescope has a 20-cm primary mirror and an active secondary mirror. Key parameters of the telescopes are given in Table 2. The telescope design is fully baffled to prevent charged particles from reaching the $\mathrm{CCD}$, and the aperture pupil is located by a mask that is mounted in front of the primary mirror. Each telescope field of view is approximately 41 arcmin circular diameter. By design, the CCD corners do not receive solar emission from the optics (being shaded by the filter-wheel mechanism). These dark regions provide useful means to monitor detector noise levels and to check for the presence of energetic particles. The telescope mirrors have multilayer coatings that are optimized for the selected EUV wavelength of interest. Three of the telescopes, numbers 1,2, and 4, have two different EUV band passes. Telescope number 3's mirror has a 171- $\AA$ band pass on one half and the other half has a broad-band UV coating. At the focal plane are back-thinned CCD sensors with $4096 \times 4096$ pixels; each $12-\mu$ m pixel corresponds to 0.6 arcsec. Entrance filters at the telescope aperture block visible and IR radiation. Filters in a filter-wheel mechanism located 
Table 1 The primary ions observed by AIA. Many are species of iron covering more than a decade in coronal temperatures.

\begin{tabular}{rlll}
\hline Channel & Primary ion(s) & Region of atmosphere & Char. $\log (T)$ \\
\hline $4500 \AA$ & continuum & photosphere & 3.7 \\
$1700 \AA$ & continuum & temperature minimum, photosphere & 3.7 \\
$304 \AA$ & He II & chromosphere, transition region & 4.7 \\
$1600 \AA$ & C IV + cont. & transition region, upper photosphere & 5.0 \\
$171 \AA$ & Fe IX & quiet corona, upper transition region & 5.8 \\
$193 \AA$ & Fe XII, XXIV & corona and hot flare plasma & $6.2,7.3$ \\
$211 \AA$ & Fe XIV & active-region corona & 6.3 \\
$335 \AA$ & Fe XVI & active-region corona & 6.4 \\
$94 \AA$ & Fe XVIII & flaring corona & 6.8 \\
$131 \AA$ & Fe VIII, XXI & transition region, flaring corona & $5.6,7.0$ \\
\hline
\end{tabular}

Table 2 The AIA instrument characteristics.

\begin{tabular}{|c|c|}
\hline Mirrors & Multilayer-coated Zerodur \\
\hline Primary diameter & $20 \mathrm{~cm}$ \\
\hline Effective focal length & $4.125 \mathrm{~m}$ \\
\hline Field of view & $\begin{array}{l}41 \times 41 \operatorname{arcmin} \text { (along detector axes) } \\
46 \times 46 \operatorname{arcmin}(\text { along detector diagonal) }\end{array}$ \\
\hline Pixel size/Resolution & $0.6 \operatorname{arcsec}(12 \mu \mathrm{m}) / 1.5 \operatorname{arcsec}$ \\
\hline CCD detector & $4096 \times 4096$, thinned, back-illuminated \\
\hline Detector full well & 150000 electrons \\
\hline Effective pointing stability (with image stabilization system) & $0.12 \operatorname{arcsec}$ RMS \\
\hline \multicolumn{2}{|l|}{ Cadence(Full-frame readout) } \\
\hline All telescopes & 8 wavelengths in 10 to 12 seconds \\
\hline Typical exposure times & 0.5 to 3 seconds \\
\hline Flight computer & BAe RAD 6000 \\
\hline \multicolumn{2}{|l|}{ Mass } \\
\hline Four telescopes & $112 \mathrm{~kg}$ \\
\hline AIA electronics box & $26 \mathrm{~kg}$ \\
\hline Main wiring harness & $17 \mathrm{~kg}$ \\
\hline Instrument power & $160 \mathrm{~W}$ \\
\hline \multicolumn{2}{|l|}{ Science Telemetry } \\
\hline Interface to spacecraft & $67 \mathrm{Mbps}$ \\
\hline Ground capture & $\approx 2$ Tbytes (uncompressed) per day \\
\hline
\end{tabular}

in front of the focal plane are used to select the wavelength channel of interest in three of the telescopes and the fourth telescope contains a selector mechanism to choose the wavelength. A mechanical shutter is used to regulate the exposure time. The active secondary is pointed in response to signals from a dedicated guide telescope (GT), which is mounted on the side of the telescope tube. The design of the AIA telescope employs many features that were successful for TRACE (Handy et al., 1999) but in comparison, each AIA telescope has 16 times more pixels than TRACE and covers the whole solar disk. AIA has sufficient 


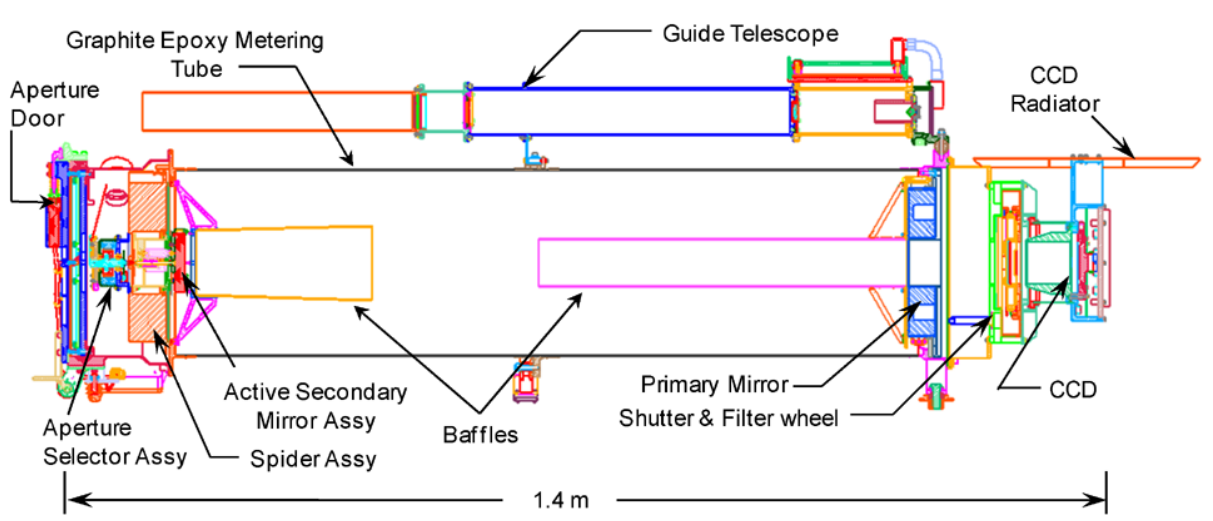

Figure 4 A cross sectional view of AIA telescope number 2 and its guide telescope. The aperture door protects the entrance filters during launch operations. Each of the four AIA telescopes has its own guide telescope, which provides a signal for the active secondary to stabilize the image on the CCD.
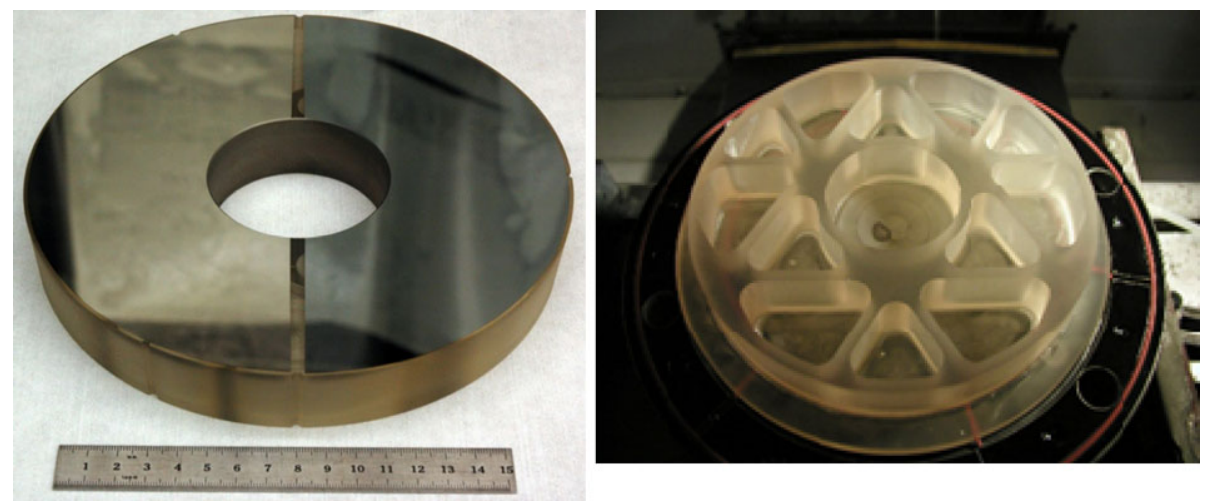

Figure 5 An AIA primary mirror (left) coated with two EUV coatings and a view of the backside of the primary mirror showing the light-weighting. The outer diameter of the primary mirror is $200 \mathrm{~mm}$ and the inner diameter is $65 \mathrm{~mm}$. The mirror is held by a low-stress mount that has flexures bonded to the outer rim of the substrate.

sensitivity to obtain a cadence that surpasses TRACE by more than a factor of ten. AIA routinely gathers as much data in three days as the TRACE mission did during its 12-year mission. A cross sectional view of AIA is provided in Figure 4. Further details about the telescope design, optics mounts, and filters are given by Cheimets et al. (2009).

\subsection{Mirrors and Multilayer Coatings}

The AIA telescope mirrors (Figure 5) are fabricated on Zerodur ${ }^{\mathrm{TM}}$ substrates, which provide a low coefficient of thermal expansion. They are figured and polished to a micro roughness of $<5 \AA \mathrm{rms}$ in the spatial frequency range from $10^{-3}$ to $5 \times 10^{-2} \mathrm{~nm}^{-1}$ as measured by atomic-force microscopy at Lawrence Livermore National Laboratory (LLNL, Soufli et al., 2007). Three of the primary flight substrates (94/304, 171/UV, and 131/335 $\AA$ ) and three of the secondary flight substrates (171/UV, 193/211, and 131/335 $\AA$ ) were fabricated by the Sagem Corporation. Sagem super-polished the substrates to a sphere, and then an ion-beam 
Table 3 Multilayer and filter properties. The metal filters are supported on a $82 \%$ transmitting nickel mesh.

\begin{tabular}{|c|c|c|c|c|c|}
\hline Telescope & $\begin{array}{l}\text { Central } \\
\text { Wavelength } \\
(\AA)\end{array}$ & $\begin{array}{l}\text { Mirror } \\
\text { Coating }\end{array}$ & $\begin{array}{l}\text { Entrance } \\
\text { Filter }\end{array}$ & $\begin{array}{l}\text { Focal-plane } \\
\text { Filter }\end{array}$ & $\begin{array}{l}\text { Redundant } \\
\text { Focal-plane } \\
\text { Filter }\end{array}$ \\
\hline 1 & 131.0 & $\mathrm{Mo} / \mathrm{Si}$ & $2000 \AA ̊ \mathrm{Zr}$ & $2000 \AA \mathrm{Zr}$ & $\begin{array}{l}3000 \AA ̊ \\
+4000 \AA \\
\text { polyimide }\end{array}$ \\
\hline 1 & 335.4 & $\mathrm{SiC} / \mathrm{Si}$ & $1500 \AA \mathrm{Al}$ & $1500 \AA \mathrm{Al}$ & $2500 \AA \mathrm{Al}$ \\
\hline 2 & 193.5 & $\mathrm{Mo} / \mathrm{Si}$ & $1500 \AA ̊ \mathrm{Al}$ & $1500 \AA ̊ \mathrm{Al}$ & $2500 \AA ̊ A l$ \\
\hline 2 & 211.3 & $\mathrm{Mo} / \mathrm{Si}$ & $1500 \AA \mathrm{Al}$ & $1500 \AA \mathrm{Al}$ & $2500 \AA \mathrm{Al}$ \\
\hline 3 & 171.1 & $\mathrm{Mo} / \mathrm{Si}$ & $1500 \AA \mathrm{Al}$ & $1500 \AA \mathrm{Al}$ & $2500 \AA ̊ \mathrm{Al}$ \\
\hline 3 & 1600 & $\mathrm{Al} / \mathrm{MgF}_{2}$ & $\mathrm{MgF}_{2}$ Window & $\mathrm{MgF}_{2}$ & - \\
\hline 3 & 1700 & $\mathrm{Al} / \mathrm{MgF}_{2}$ & $\mathrm{MgF}_{2}$ Window & Fused silica & - \\
\hline 3 & 4500 & $\mathrm{Al} / \mathrm{MgF}_{2}$ & $\mathrm{MgF}_{2}$ Window & Fused silica & - \\
\hline 4 & 93.9 & $\mathrm{Mo} / \mathrm{Y}$ & $2000 \AA ̊ \mathrm{Zr}$ & $2000 \AA \mathrm{Zr}$ & $3000 \AA ̊ \mathrm{Zr}$ \\
\hline 4 & 303.8 & $\mathrm{SiC} / \mathrm{Si}$ & $1500 \AA \mathrm{Al}$ & $1500 \AA \mathrm{A} 1$ & $2500 \AA \mathrm{Al}$ \\
\hline
\end{tabular}

etch was used to achieve the desired asphere, while preserving the required super-polished surface. L-3 Communications-Tinsley Laboratories produced the flight primary (193/211 $)$ and secondary (94/304 ̊̊) substrates using a classic, computer-controlled grind and superpolishing approach.

Different multilayer coatings were applied to each half of each telescope optic to achieve the desired central wavelength (see Table 3). Reflective X-ray Optics applied the 94-, 131-, and 335- $\AA$ flight coatings and LLNL applied the 171-, 193-, 211- $\AA$ flight coatings (Soufli et al., 2005). LLNL led the reflectance calibration and mapping of all AIA EUV flight mirrors, using the reflectometer at beam line 6.3.2 of the Advanced Light Source synchrotron at Lawrence Berkeley National Laboratory (Underwood et al., 1997; Gullikson, Mrowka, and Kaufmann, 2001).

The UV channel in AIA telescope 3 is based on the TRACE design and makes use of a $\mathrm{MgF}_{2}$ window with a coating centered at $1600 \AA$. The mirrors were coated by the Acton Research Corporation (now a division of Princeton Instruments) with aluminum with an $\mathrm{MgF}_{2}$ protective overcoat. Three filters in the telescope number 3 filter wheel, also manufactured by Acton, select the band passes of interest: $1600 \AA$ on $\mathrm{MgF}_{2}$, which includes the C IV lines, $1700 \AA$ on fused silica, which suppresses the C IV lines, and a $\approx 500$ - $\mathrm{A}$ FWHM band pass filter centered at $4500 \AA$.

The mechanical mounts of the mirrors were designed to maintain arc-second alignment during launch and to ensure that thermally induced stresses during on-orbit operations do not introduce aberrations that would degrade the imaging performance (Podgorski et al., 2009). The primary-mirror mount contains titanium flexures that are bonded to the mirror in three locations on the side of the substrate. The flexures are attached to a titanium ring that is the interface to the aft end of the metering tube. The mount design can withstand temperature changes of up to $\pm 12^{\circ} \mathrm{C}$ without compromising the imaging performance. The secondary mirror is clamped in three locations and is pressed by a spring onto three PZT actuators that provide imaging stabilization. 


\subsection{Filters}

The AIA design makes use of metal entrance filters at the aperture of each telescope and in a filter wheel located in front of each focal plane. The filters suppress unwanted UV, visible, and IR radiation for the EUV channels. Two filter materials are used for the filters, aluminum and zirconium, whose transmissions are illustrated in Figure 2 of Boerner et al. (2011). Aluminum is used for $171 \AA$ and longer wavelengths and zirconium is used for the two shorter wavelengths. Because of the filter-transmission properties, the selection of aluminum or zirconium in the filter wheel is sufficient to select between the shorter or longer band pass in AIA telescope number 1 , which contains $131 \AA$ and $335 \AA$, and telescope number 4 , which contains $94 \AA$ and $304 \AA$. The redundant, thicker zirconium filter in telescope number 1 also includes a 4000 - $\AA$ layer of polyimide to provide the option of additional attenuation at $131 \AA$ during very bright flares. The filters and their respective thicknesses are given in Table 3. Each focal-plane filter contains redundant thicker filters in case the thin filters develop pinholes. The filters are supported on a nickel mesh with a 70 line-per-inch spacing. The mesh of the front filter results in a diffraction pattern that is visible in high-contrast scenes such as solar flares (Lin, Nightingale, and Tarbell, 2001). The mesh in the focal-plane filters results in a faint shadow pattern in the focal plane, which is removed by flat fielding. All metal filters were manufactured by Luxel Corporation. The focal-plane filter transmissions were measured in Lockheed Martin Solar and Astrophysics Laboratory's (LMSAL) calibration facility (Boerner et al., 2011). Entrance-filter transmissions are computed using Luxel Corporation-provided filter-material thicknesses.

\subsection{CCD Detector and Camera System}

The AIA and HMI instruments use CCDs that have the same architecture. This enables the camera-electronics boxes to be interchangeable between the two instruments. The CCDs are custom designed e2v technologies CCD203-82 devices, the AIA ones are back-thinned and back-illuminated while the HMI detectors are front-illuminated. The CCDs are operated non-inverted to obtain good full-well capacity $\left(>150 \mathrm{ke}^{-}\right.$pixel $\left.^{-1}\right)$. The pixel size is $12 \mu \mathrm{m}$ and each detector has $4096 \times 4096$ pixels (Figure 6). There are four readout amplifiers on each device and during normal operations, all four $2048 \times 2048$ quadrants are read out simultaneously. The quantum efficiencies of the AIA CCDs have been characterized in the laboratory at LMSAL (Boerner et al., 2011). Laboratory exposure-dose tests conducted at $304 \AA$ revealed modest reduction in quantum efficiency with high dosage. This slight reduction is partially reversible by warming the device, consistent with the presence of trapped charge in the oxide layer.

The camera electronics (Figure 7) were developed at the Rutherford Appleton Laboratory (Waltham et al., 2011). The design is based on previous flight designs, most recently for the cameras of the STEREO/SECCHI instrument (Howard et al., 2008). There are four AIA camera-electronics boxes, one for each telescope, and each camera has four interfaces to read out simultaneously the signals from the four CCD amplifiers. Each quadrant is read at a rate of 2 Mpixels s ${ }^{-1}$ with less than 25 electrons of read noise. The camera implements correlated double sampling and analog-to-digital functions in an RAL-designed, radiation-hardened ASIC. Each camera communicates with the AIA electronics box by an IEEE1355/SpaceWire link, which enables data transmission rates of up to $100 \mathrm{Mbits} \mathrm{s}^{-1}$. The camera has internal dedicated DC-DC power converters to convert the SDO spacecraft $28 \mathrm{~V}$ primary power to the required voltages. The camera has programmable control of the 
Figure 6 A front-illuminated CCD203-82 device mounted in its nickel-plated Invar 36 package. The AIA flight-version CCD is back-thinned and back-illuminated.
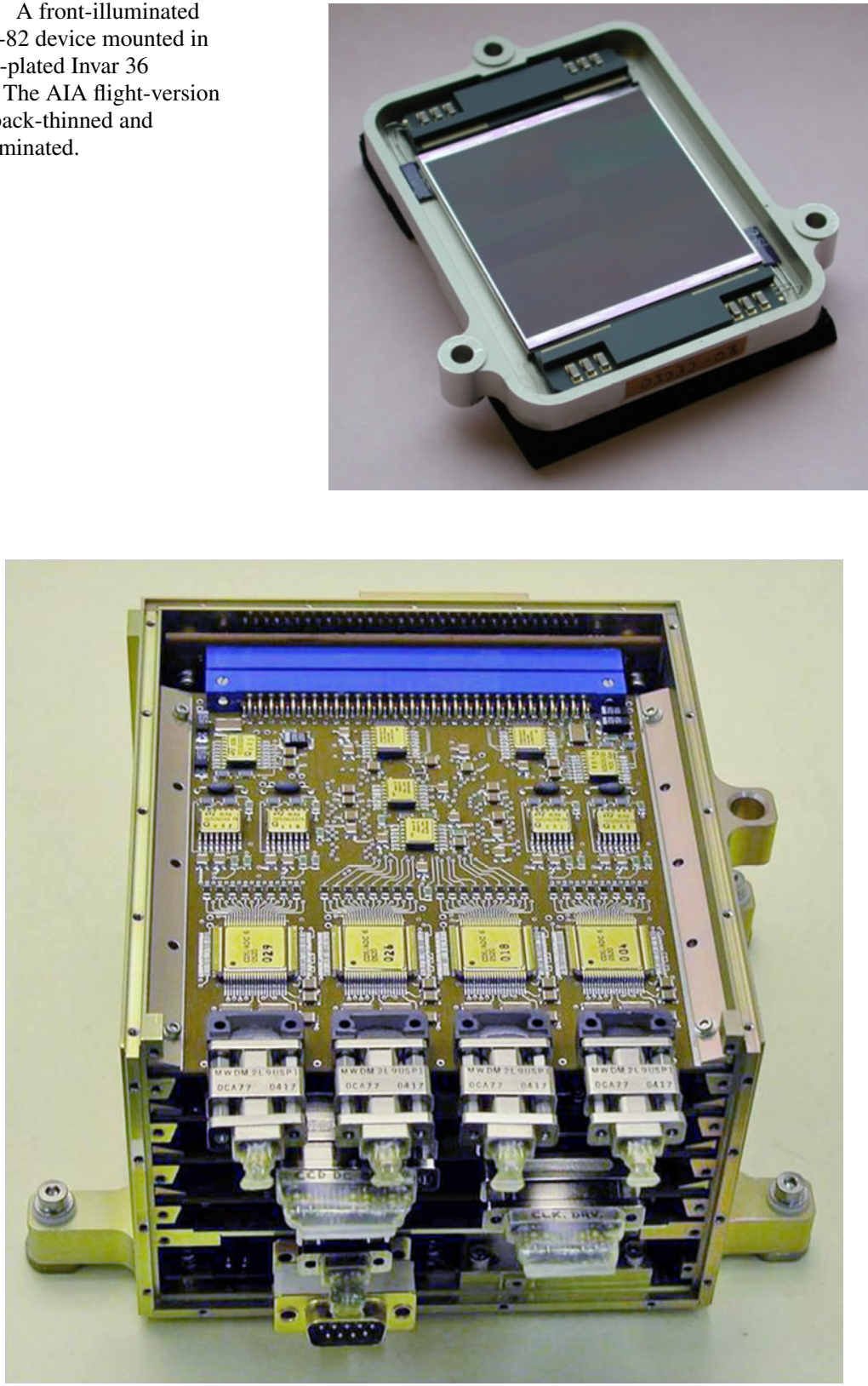

Figure 7 The camera-electronics box (CEB) shown is used in both the AIA and HMI instruments. AIA contains four cameras and HMI two. The camera can operate at up to 2 Mpixels s $^{-1}$ and is interfaced to the AIA electronics box via a SpaceWire link.

waveform generator to provide different operating modes - such as continuous readout, fullframe or windowed, clearing, summing, and exposure - and it provides on-orbit adjustment of gain and pedestal offset. 
Table 4 Key Parameters of the AIA Guide Telescope and Image Stabilization System
Figure 8 The Guide Telescope shown mounted on the AIA number 2 telescope.

\begin{tabular}{ll}
\hline Wavelength and band pass width & $570 \mathrm{~nm}, 50 \mathrm{~nm}$ band \\
Focal length & $1665 \mathrm{~mm}$ \\
Linear range of GT & $> \pm 95 \mathrm{arcsecs}$ \\
Acquisition range & $1570 \mathrm{arcsec}$ \\
Noise equivalent angle & $<0.12 \mathrm{arcsec}$ \\
ISS mirror range & \pm 46 arcsec (in AIA telescope \\
& focal plane) \\
Low frequency jitter reduction factor & 50 \\
ISS bandwidth (cross-over) & $30 \mathrm{~Hz}$ \\
\hline
\end{tabular}

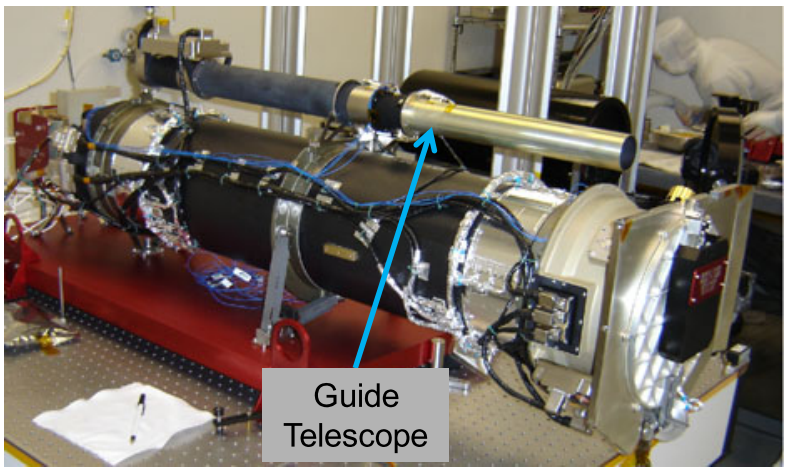

Decontamination heaters are attached to each CCD through a switch controlled by the spacecraft electronics. The CCD heaters were activated shortly after launch for a monthlong instrument bakeout. They will be used periodically during the SDO mission to clear the buildup of contaminants as necessary and to eliminate trapped charge in the oxide layers.

\subsection{Guide Telescope and Image-Stabilization System}

Each EUV telescope has its own guide telescope (GT) which provides an error signal to its image stabilization system (ISS). The GT is based on the TRACE GT and STEREO/SECCHI designs and consists of an achromatic refractor with a band-pass entrance filter that is centered at $570 \mathrm{~nm}$ and a Barlow-lens assembly. Four redundant photodiodes positioned behind an occulter measure the position of the solar limb. Unlike TRACE, the AIA/GT has no prisms to off-point the Sun because the AIA instrument has a full-disk field of view. Other differences compared to TRACE include increased shielding for the preamplifier and diodes, and radiation-hardened glass for the entrance window. The key parameters for the GT are shown in Table 4. The GT mounted to telescope number 2 is shown in Figure 8.

The signals for the diodes are amplified and provided to the limb-tracker electronics in the main AIA electronics box (AEB) which in turn controls the three piezoelectric transducers (PZTs) that activate the secondary mirror. Spacecraft jitter in pitch and yaw is reduced to less than $0.24 \operatorname{arcsecs}(1 \sigma)$ in each axis and is effective for frequencies less than $\approx 10 \mathrm{~Hz}$. Each PZT can tilt the secondary mirror by \pm 46 arcsecs in the telescope focal plane (i.e., in the image). Like TRACE, the ISS has gain adjustments that may be calibrated on orbit from data taken while slewing the observatory across the solar limbs. Calibration observations are planned to be accomplished monthly. 
Figure 9 The aperture selector mounted on the telescope number 2 secondary mirror spider assembly. The focus motor can be seen on the left.

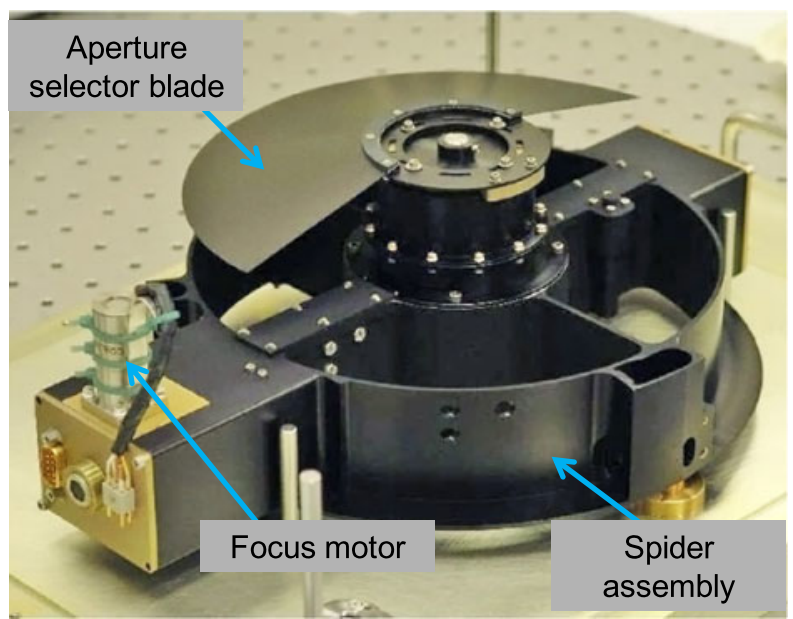

The AIA flight software samples the GT signals every $4 \mathrm{~ms}$ with a 12-bit ADC and this signal is provided to the SDO spacecraft attitude control system (ACS) software to provide a fine Sun-sensor signal. Any one of the GTs may be selected as the sensor input for the spacecraft ACS. If all four diodes are illuminated, then the GT is in its linear range and the error signals are valid. If fewer than four diodes are illuminated, then Sun-presence flags are set for the appropriate diodes and these may be used by the ACS to acquire the Sun.

\subsection{Mechanisms}

AIA contains a total of 17 mechanisms consisting of five distinct types. Each telescope contains a front door, a focus mechanism, a filter wheel, and a shutter mechanism. Telescope number 2 also contains an aperture selector.

The front door is designed to protect the entrance filters during launch from debris and from acoustic loads. The AIA design shares heritage with the TRACE and Hinode/XRT designs. The door is latched shut with a high-output paraffin actuator which has redundant heater circuits. A Viton O-ring ensures a good seal when closed. The door hinge is springloaded and the door is slightly bowed to promote the 180-degree opening when on orbit. Although the paraffin actuators may be used multiple times, there is no means to close the door once on orbit, so it is considered a "one-shot" operation. All four of the AIA doors deployed successfully at the end of the bake-out period, approximately one month after launch.

AIA telescope number 2 has an aperture selector which enables only one half of the aperture to be exposed at one time. The aperture selector blade is $211 \mathrm{~mm}$ in diameter and is required to select between $193 \AA$ and $211 \AA$ (Figure 9). In the other telescopes, the selection of the filter in the filter wheel can be used to select the desired channel, but since both of these band passes require aluminum filters, the aperture selector was necessary. The Kollmorgen RB-01501 motor can move from one position to the other in less than a second. This approach is the same as was employed for TRACE and STEREO/SECCHI. This mechanism and all the other mechanisms (apart from the door) were life tested in a vacuum for over 60 million operations, approximately double the number expected during the five-year prime SDO mission.

Each telescope contains a focus mechanism that adjusts the position of the secondary mirror relative to the primary along the optical axis by up to $\pm 800 \mu \mathrm{m}$ in $2.2-\mu \mathrm{m}$ steps. As 
Figure 10 The AIA filter-wheel mechanism. The wheel has five openings containing various filters for each telescope as described in Table 3.

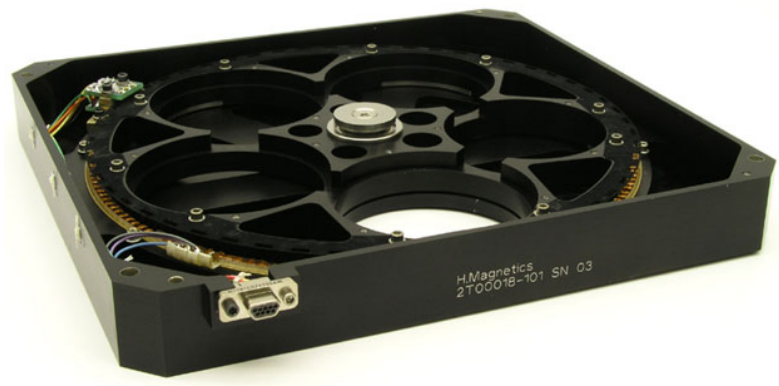

Figure 11 The AIA shutter mechanism consisting of its blade and shutter motor. The narrow slot is used for 5-ms exposures and the larger opening is used for 80-ms and longer exposures.

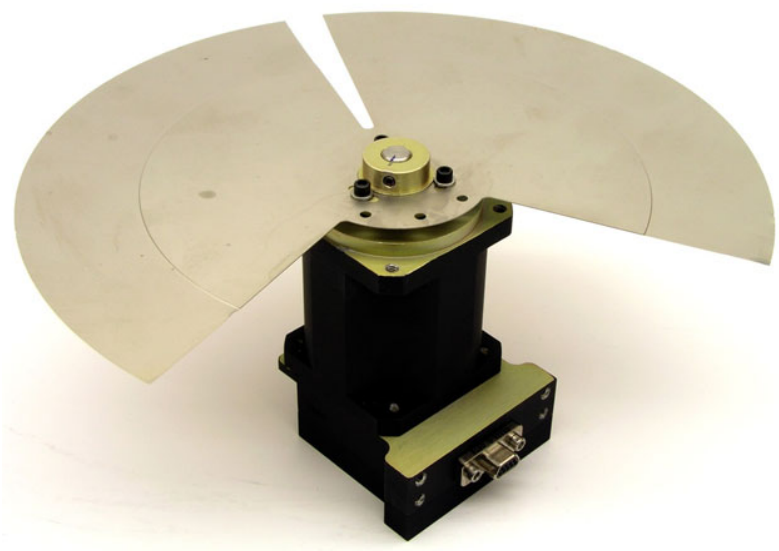

the optical design has a magnification of three, this corresponds to an approximately $22-\mu \mathrm{m}$ change in focus per step. The design is based on the TRACE focus mechanism and employs a stepper motor manufactured by CDA InterCorp to drive a screw that pushes a level arm attached to the secondary mirror cell. The focus can be adjusted as often as every exposure if necessary.

The filter wheel is based on the Triana/Earth Polycromatic Imaging Camera (EPIC) and Hinode/XRT designs and consists of a thin, brushless DC motor manufactured by H. Magnetics (Figure 10). To the motor is added an optical encoder, and the mechanism is operated as a stepper motor with 324 steps per revolution. A one-position move requires about one second. For telescope numbers 1, 2, and 4 the filter wheel contains four metal (focal-plane) filters and the fifth position is left open for calibration purposes. Telescope number 3 contains two metal filters and three glass filters (for the UV channels).

The shutter mechanism consists of a $159-\mathrm{mm}$ diameter thin blade mounted to a Kollmorgen RB-00704-G02 motor (Figure 11). The housing and optical encoder are a LMSAL design and the bearings are from Timken MPB. This design has flight heritage on several missions including SOHO/MDI, TRACE, STEREO/SECCHI, and Hinode/Focal Plane Package (FPP) and XRT. The blade rotates to open the shutter, exposing the CCD. The blade has a narrow opening that provides a 5-ms exposure when rotated over the CCD and a wide opening that provides an 80-ms exposure. Longer exposures are obtained by stopping the wide opening in front of the CCD for a specified length of time that is controlled by the flight software. Exposure times longer than $5 \mathrm{~ms}$ and shorter than $80 \mathrm{~ms}$ are acquired by sweeping the shutter's narrow opening over the CCD multiple times. 


\subsection{Electronics and Software}

The AIA electronics shares much of its design with HMI and has selective redundancy. The AIA electronics box (AEB) provides power and housekeeping (MIL-STD-1553) interfaces to the spacecraft in addition to the science-data interface by way of two redundant IEEE 1355 SpaceWire ports. The AEB contains redundant DC-DC power systems and redundant RAD6000 CPU cards, the latter being procured from BAE Systems. A block diagram of the electronics system is shown in Figure 12.

The flight software was developed using the real-time multi-tasking VxWorks environment. The software is responsible for receiving commands from the spacecraft and controls the flow of housekeeping data and science data to the spacecraft. It also is responsible for the interfaces to the mechanisms, the heaters, the guide telescope, and the cameras.

The image sequencer controls the scheduling of images in the four telescopes and is configured by tables that can be uploaded from the ground. Under typically planned operations, the basic time step is set to 12 seconds and each telescope is able to acquire two images within each 12-second window, and so eight full-images are acquired every 12 seconds. Because the four cameras share two interfaces to the spacecraft, there are various constraints on how long an exposure can be in a particular telescope. Under nominal operations exposures up to 2.9 seconds are possible. The sequencer design is flexible to support the AIA science observing objectives and is very robust. It is inherently deterministic, provides accurate temporal scheduling, and was the basis for the HMI sequencer design. The flight software has the TRACE automatic exposure-control algorithm implemented to enable the control of exposure times in case of flares.

\subsection{Instrument Calibration}

The AIA calibration is described by Boerner et al. (2011). The instrument effective area has been estimated from component-level calibration measurements. Responses to solar emissions are computed assuming the CHIANTI solar spectral model (Dere et al., 1997, 2009). The response functions for the six EUV band passes that are dominated by iron emission lines are shown in Figure 13. One of the channels in telescope 4 is centered on the emission from He II at 303.8 $\AA$. This band also contains emission from nearby Si XI at $303.3 \AA$, which may contribute up to $20 \%$ of the total intensity detected in this channel when observing active regions (Thompson and Brekke, 2000). AIA calibration data and computed response functions may be accessed on-line (see Boerner et al., 2011) to facilitate detailed or specific analyses.

\section{Instrument Operations}

The AIA is designed to operate in a regular, synoptic fashion, and it is anticipated that the observing program will be changed infrequently. The initial baseline observing program acquires a full-frame EUV image and one UV or visible-light image every 12 seconds. The basic cadence can be adjusted and may be shortened to 10 seconds if warranted by active solar conditions. The AIA camera electronics support the read out of small regions of interest, which reduces the observing cadence to 2 seconds for selected wavelength channels in order to achieve special campaign observing objectives.

At the baseline 12-second cadence, the data acquisition exceeds the AIA telemetry allocation by a factor of 2.2, thus necessitating the use of on-board compression. AIA has two 


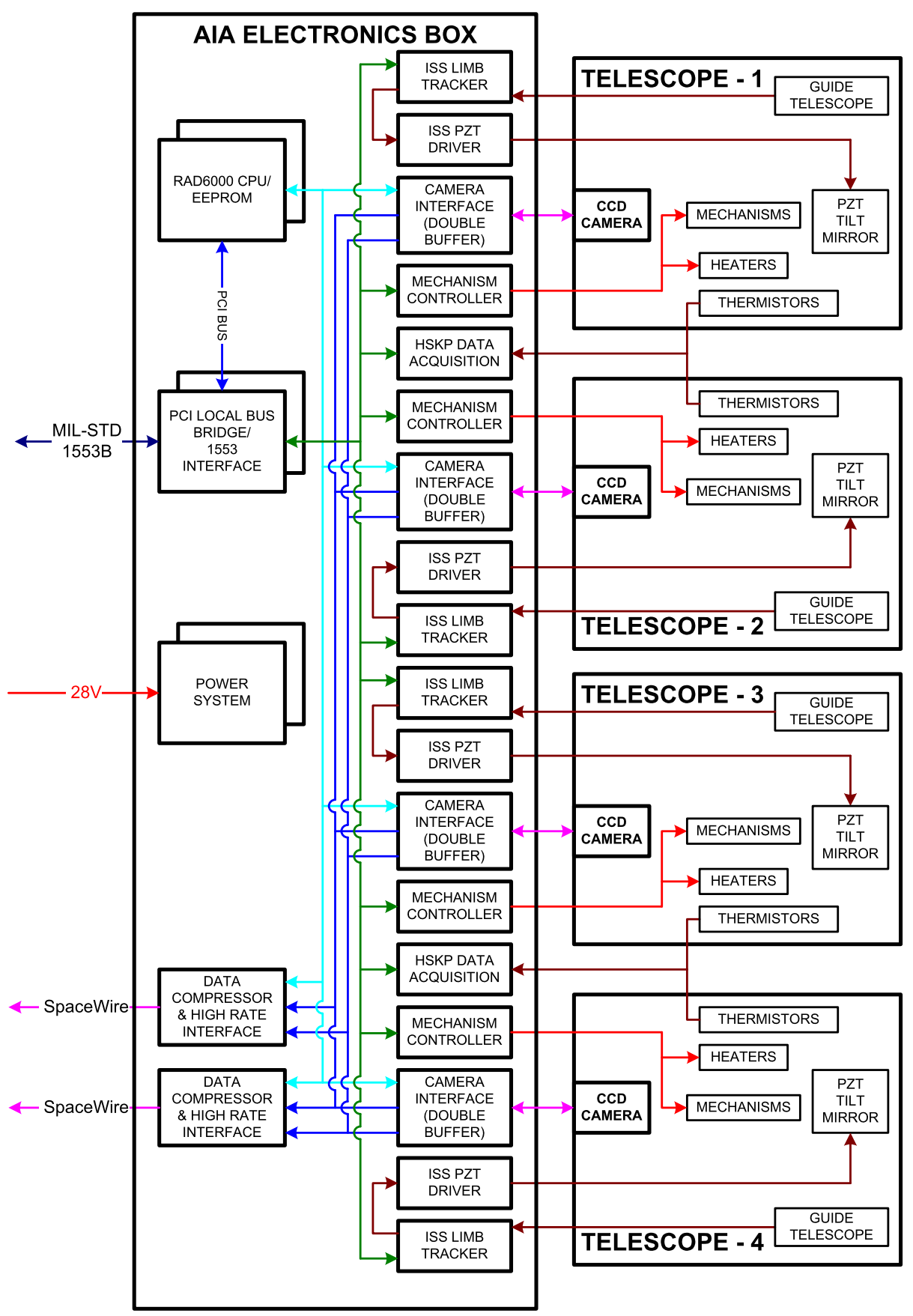

Figure 12 The AIA electronics block diagram. The AIA electronics box interfaces include the spacecraft (to the left) and the four AIA telescopes (to the right). 


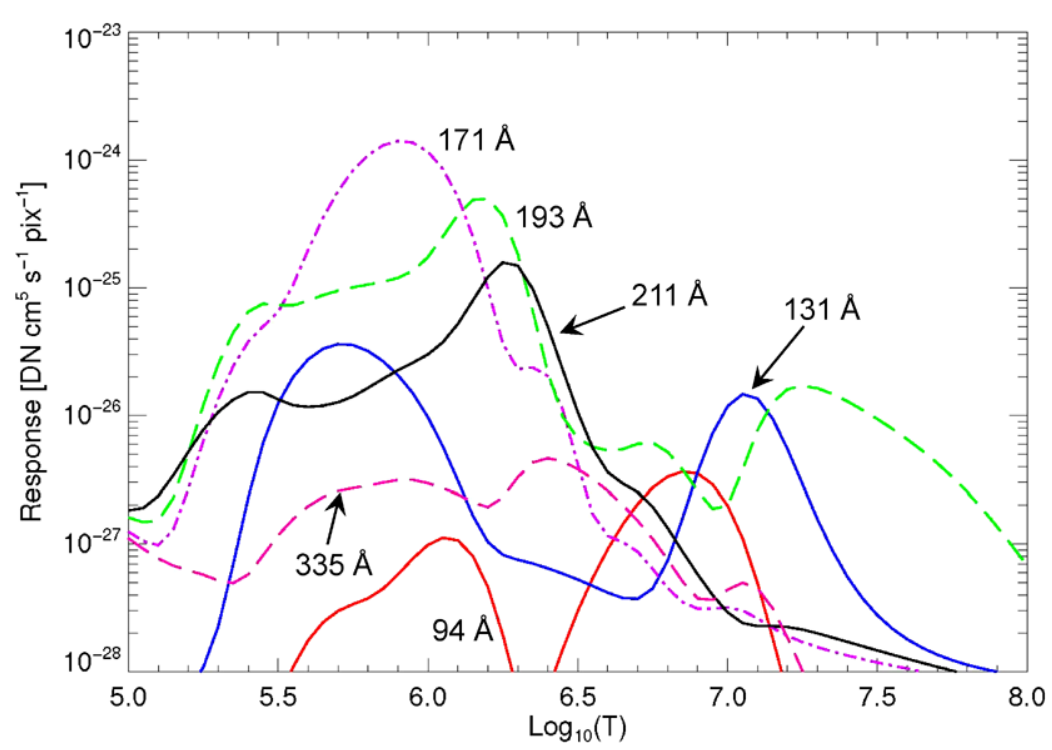

Figure 13 Temperature response functions for the six EUV channels that are dominated by iron emission lines calculated from the effective-area functions and assuming the CHIANTI model for the solar emissivity. This figure corresponds to Figure 11 of Boerner et al. (2011) and Figure 8 of Boerner et al. may be consulted for the corresponding effective-area functions.

data compression/high-speed interface cards that perform data compression in hardware and then transmit the compressed data to the spacecraft interface. Two compression algorithms are implemented in field-programmable-gate arrays whose parameters can be fine-tuned depending on the wavelength channel. The primary algorithm is lossless Rice compression, which typically achieves a factor of two reduction in data volume for EUV images. A nonlossless algorithm relies on a look-up table that is configurable from the ground. The baseline table is a scaled square-root function. More details are provided by Boerner et al. (2011). For the baseline 12-second observing mode, the longest exposure that can be acquired is limited to 2.9 seconds as 3.1 seconds is required for camera readout and other system overhead, and then this repeated in the next 6 seconds for the other companion telescope channel.

An automatic exposure control is implemented similar to that which is used on TRACE. A count-rate histogram is acquired in the hardware on the data compression/high-rate interface board prior to compression. The flight software retrieves the histogram and quickly analyzes whether the next exposure time should be made larger or smaller. Shorter exposure times during impulsive flares helps to reduce saturation caused by the acquired signal exceeding detector full well.

Science operations for both AIA and HMI are controlled from the Instrument Operations Center at LMSAL. Tables are prepared that configure the observing sequence and other instrument parameters, and are transmitted on a secure Internet connection to the SDO mission operations center at GSFC before being transmitted to the spacecraft. Routine operations typically require only a few command loads during the work week. Special operations to establish calibration parameters for telescope alignment, focus, and ISS are scheduled to occur periodically. 


\section{Data Processing}

The SDO mission does not record science data on orbit, but rather transmits telemetry continuously to two SDO ground stations located near White Sands, New Mexico. From there, the AIA and HMI data are transferred by dedicated broad-band circuits to the Stanford University campus where the Level 0 data are permanently archived in the Joint SDO Operations Center (JSOC) science-data processing (SDP) facility. Telemetry data typically arrive at the JSOC-SDP minutes after they are acquired on orbit. If a problem in ground transmission occurs, the retransmission is expected to occur within one to two days. A local storage at the SDO ground system in New Mexico has 30 days of storage as a contingency for shortterm problems with the network or at the JSOC-SDP. AIA images are assembled from the telemetry packet data and decompressed, and then stored using lossless Rice compression. Instrument and spacecraft housekeeping data are received in various telemetry packets and these are decoded and archived in the JSOC-SDP for use in later data processing. These data as archived in the SDP are considered the permanent HMI and AIA data archive and are archived onto 800 Gbyte tapes with a duplicate stored at LMSAL.

The data recorded at the JSOC-SDP are labeled as Level 0. Parallel data pipeline computing systems process the HMI and AIA Level 0 data to produce Level 1 and higher data products. The processing steps to produce Level 1 and Level 1.5 AIA data are described below. Level 1.5 includes a correction for plate scale, roll, and telescope coalignment. The data record management system (DRMS) provides a sophisticated and efficient system to retrieve and distribute AIA data and associated metadata to co-investigators and to the solar community.

\subsection{Level 1 Data Processing}

Level 0 images are stored in the data archive as 16-bit integer $4096 \times 4096$ arrays, even if only a portion of the CCD is read by the camera. The data are Rice compressed in order to reduce the ground storage requirements. Processing Level 0 data to Level 1 involves several steps. If the camera readout format includes "over-scan" rows and columns, these are removed. The over-scan rows and columns are not real, but contain the charge remaining after 2048 parallel transfers (for one quarter of the CCD) or 2048 serial transfers, and thus provide a means by which CCD charge transfer efficiency may be monitored on orbit. It is planned to only occasionally include over-scan rows and columns in AIA operations for CCD engineering purposes.

Step 2 is the removal of a dark image to account for the digital offset of the camera, CCD read noise, and dark current. The on-orbit temperature of the CCDs is typically less than $-65^{\circ} \mathrm{C}$, and thus dark current is negligible for images with exposures of a few seconds or less.

Step 3 applies a flat-field correction to the AIA images. A flat-field image is constructed for each telescope channel to account for detector non-uniformities, gain differences between CCD quadrants, vignetting, and shadowing caused by the focal-plane filter nickel support mesh. The detector non-uniformities are determined from multiple images acquired on orbit with different solar pointing coordinates, so that the resulting flat-field image represents only the instrumental effects. These are routinely updated because the AIA back-illuminated CCD silicon surface is not completely passivated and the CCD charge-collection efficiency drops as charge produced by observed solar emissions is trapped in the device oxide layer. Ground testing has demonstrated that significant restoration of sensitivity is possible by warming the CCD, and this is planned for on-orbit operations. Thus, new flat-field images 
will always need to be constructed following such CCD warming operations, and the Level 1 processing keeps track of the appropriate flat-field data to use, depending on the epoch of the acquired data that is being processed.

Step 4 makes corrections to individual pixels according to two different algorithms. The first algorithm corrects permanently "bad" pixels that do not respond correctly to light. Each CCD has a small number of such pixels, much less than $0.1 \%$, and their values are replaced with interpolated values from neighboring pixels. As the SDO mission progresses, we expect that there will be additional permanently bad pixels. Thus, the processing pipeline will maintain a time-dependent log of bad pixels for each CCD so that older data can be reprocessed correctly using the appropriate bad pixel list for the epoch of the acquired data. The second algorithm seeks to remove the "spikes" that appear as the result of the interaction of energetic particles that either deposit energy directly in the CCD or interact with the instrument structure and give rise to high-energy photons that are detected in the CCD. The spikes typically look like isolated features that can be as small as one pixel or may in some cases leave a streak of deposited energy in many adjacent pixels. The AIA "despike" algorithm was adopted from one used by the TRACE program. Spikes are detected by comparing the value of each pixel with that of neighboring pixels and flagging occurrences where the differences are not statistically consistent with what is expected given the telescope pointresponse function. Once the "spikes" are found, they are replaced with the median value of neighboring pixels. The addresses of these pixels and their original values are stored separately so that the algorithm can be easily reversed. For example, for the study of very faint features or for very compact flares it may be desirable to omit the despiking algorithm, as the procedure may introduce undesirable artifacts in the final images. The approach adopted for the pipeline processing makes it possible to "undo" the despiking step.

In Step 5 the AIA images are flipped so as to put solar North at the top of the array. The metadata are updated to add the required information about the image and a small amount of spacecraft housekeeping data is included, the most important being the roll angle. The attitude information is consistent with the world coordinate system (Thompson, 2006). The camera gain $\left(\mathrm{e}^{-} \mathrm{DN}^{-1}\right)$ and the effective area (Boerner et al., 2011) are included with the metadata.

Much of the information used to process AIA data to Level 1 depends on calibration data or algorithms that are time dependent. These include the camera gain $\left(\mathrm{e}^{-} \mathrm{DN}^{-1}\right)$, effective area, pointing information, bad-pixel list, flat field, and average band pass effective area. Information is included with the metadata to identify the algorithm or calibration data used to produce the Level 1 data products. As newer calibration data becomes available, it is possible to reprocess the Level 1 data using the most recently available calibrations.

Level 1 data are typically exported from the JSOC as FITS files with the data represented as 32-bit floating values. If it is desired to reverse the despiking procedure it is also necessary to request the list of corresponding despike pixels addresses and their original data values.

The Level 1 processing produces calibrated AIA data in all respects except for the deconvolution of the instrument point spread function, which is made up of the contributions from the mirrors and the CCD, and from the diffraction caused by the entrance filters. As described in Section 3.1, the entrance filters are either aluminum or zirconium layers supported by a 70 line-per-inch nickel square-pattern mesh. EUV light passing through these filters is diffracted by the mesh. The pattern of the diffraction is best seen when the intensity is high, such as for a flare, and has been observed with TRACE (Lin, Nightingale, and Tarbell, 2001). The diffraction pattern depends on the wavelength of the light, its intensity, the spacing of the mesh bars, the thickness of the bars, and the rotation angle of the mesh with respect to the focal plane. Each EUV channel has two filters in its aperture. The filters 
are oriented so that the mesh is rotated approximately $40^{\circ}$ and $50^{\circ}$, respectively, relative to the CCD rows and columns. So the diffraction patterns can be separated for each filter and deconvolved from the image, since the diffraction patterns themselves are well known. The omission of this step primarily effects the presentation of flare images that have bright features.

\subsection{Level 1.5 Data Processing}

The processing of Level 1 data is sufficient to produce calibrated AIA images. However, a key advantage of AIA over previous missions is high temporal cadence, full-solar-disk images in multiple wavelength channels. Thus, it is important to be able to display the AIA images as a movie sequence. Level 1.5 processing accomplishes three things that enable the display of AIA movies.

Level 1.5 processing starts with Level 1 data and applies three corrections in a single processing step. The images are rotated so that solar North is at $0^{\circ}$ (at the top of the image array). The four AIA telescopes were aligned prior to launch but no further adjustment is available on orbit, so a small residual roll angle exists between the four AIA telescopes. This residual roll is removed and the spacecraft star-tracker signal is used to place solar North at $0^{\circ}$. The second correction has to do with the plate-scale size of the telescopes. Each telescope has a slightly different focal length; as a result, the number of arcsecs per CCD pixel varies slightly between the telescopes. A plate-scale adjustment is made to set the image scale to exactly 0.6 arcsecs per pixel. If it becomes necessary to refocus a telescope during the mission, the plate-scale calibration will be repeated to ensure that the Level 1.5 processing produces the correct plate scale. Finally, as with the roll angle, the bore-sight pointing of each telescope was coaligned prior to launch to within 20 arcsec. The post-launch measurements indicate that the alignment was not significantly changed by the launch activities. The four telescope bore-sights are coaligned by adjusting on orbit the secondary-mirror offsets. Residual differences are removed during ground processing by interpolating the images onto a new pixel coordinate that places the Sun's center at the middle of each telescope detector. The data processing pipeline implements a damped bi-cubic interpolation algorithm (Park and Schowengerdt, 1983) to make plate-scale, roll, and alignment adjustments. Intensities are rounded off and saved as 16-bit integer values. Those who wish to preserve fractional intensity values may optionally process Level 1 with routines available through SolarSoft (Freeland and Bentley, 2000).

As with the parameters used for the Level 1 processing, small time-dependent changes in the plate-scale and alignment parameters are anticipated during the life of the mission, mostly due to orbital effects that cause variations in the distance to the Sun and thermal variations of the telescopes. Calibration data will be regularly acquired and the Level 1.5 processing will access the calibration parameters appropriate to the epoch of data that is processed.

Along with the processing actions described above, the associated metadata is updated to reflect the final Level 1.5 calibrated state. Because of the nature of these processing steps, the operation of processing Level 1 to Level 1.5 is not reversible. When exported as FITS files, the file header structure for Level 1 and Level 1.5 have exactly the same keywords but with values set to reflect the level of processing. HMI magnetograms are provided along with AIA Level 1.5 data. The magnetograms are rescaled down to the AIA plate scale of 0.6 arcsec (which reduces the HMI resolution slightly) and the roll and alignment are adjusted to be the same as AIA. 
Table 5 Useful links for AIA data, events, and analysis documentation.

\begin{tabular}{ll}
\hline Item & URL \\
\hline Analysis user's guide & www.Imsal.com/sdodocs/public_docs/sdo_analysis_guide.html \\
General documentation & www.Imsal.com/sdodocs \\
Recent AIA Images & sdowww.Imsal.com/suntoday \\
Sungate portal & www.Imsal.com/sungate \\
\hline
\end{tabular}

\subsection{Data Distribution}

The processed AIA data are made freely available for public access approximately 48 hours after receipt at the JSOC. The large volume of Level 1 and 1.5 data products are transferred to the Smithsonian Astrophysical Observatory to act as a data-distribution center for scientists around the world. Many researchers will make use of the Virtual Solar Observatory (VSO), which has developed a data-distribution architecture that is consistent with the JSOC. The Heliophysics Event Knowledgebase (Hurlburt et al., 2011) will aid users in their search of the SDO event database and also facilitate efficient data retrieval. Additional ancillary databases are made available in the SolarSoft database distribution. Further information about SDO data and database distribution systems are available at the links provided in Table 5.

\section{Conclusion}

The AIA doors were opened and first images were acquired on 27 March 2010. Since then AIA has returned TRACE-like high-resolution images, but with a field of view that covers the entire solar disk in seven EUV wavelength channels and with an unprecedented cadence. AIA movies reveal a fascinating level of structure and variability in the solar atmosphere. The full-disk, high-resolution observations reveal that even minor flare and filament eruptions and disturbances are related to global magnetic restructuring.

In addition to the seven EUV channels, AIA also observes in two UV channels near 1600 and $1700 \AA$ A. A visible-light channel provides a means for coalignment with other observatories. All four telescopes are well coaligned with each other and their individual image-stabilization systems are working as expected, to eliminate jitter produced by the spacecraft. Except for two periods of the year near the Vernal and Autumnal Equinoxes, AIA is able to observe almost continuously (more than $95 \%$ of the time) due to the dedicated ground station in White Sands, New Mexico. The regular synoptic observing sequence can be adjusted, but is typically operated a cadence of 10 or 12 seconds. The flight software has flexibility to enable seasonal and long-term refinements to the observing program.

After nearly a year on orbit the spacecraft and the AIA are functionally extremely well. There is an excellent probability that the AIA performance will exceed its five-year prime mission lifetime. All of the SDO data are available to the public without restriction.

Acknowledgements The effort required to build an instrument such as AIA requires a large, skillful, and dedicated team. We wish to acknowledge many individuals who contributed to the success of the AIA: Robert Batista, Roger Chevalier, Dustin Cram, Cliff Evans, Scott Gibb, Dwana Kacensky, Robert Honeycut, Bruce Imai, Alex Price, Lawrence Shing, Edgar Thomas, Shanti Varaich, Ross Yamamoto, Kent Zickhur (Lockheed Martin), Gerald Austin (Smithsonian Astrophysical Observatory), David McKenzie (Montana State Univ.), Eberhard Spiller, Jeff C. Robinson, Sherry L. Baker (Lawrence Livermore National Lab.), Travis Ayers, Heidi 
Lopez, Forbes Powell, (Luxel Corp.), and Tom Anderson, Elizabeth Citrin, Julie Lander, Chad Salo, and Mike Scott (NASA Goddard Space Flight Center). Lawrence Livermore National Laboratory's efforts are partially supported by the U.S. Department of Energy under contract DE-AC52-07NA27344. This work is supported by NASA under contract NNG04EA00C and the Lockheed Martin Independent Research Program.

\section{References}

Boerner, P., Edwards, C., Lemen, J., Rausch, A., Schrijver, C., Shine, R., Shing, L., Stern, R.A., Tarbell, T., Title, A., Wolfson, C.J., Gullikson, E., Soufli, R., Spiller, E., McKenzie, D., Windt, D., Golub, L., Podgorski, W., Testa, P., Weber, M.: 2011, Solar Phys., accepted.

Cheimets, P., Caldwell, D.C., Chou, C., Gates, R., Lemen, J., Podgorski, W.A., Wolfson, C.J., Wuelser, J.-P.: 2009, Proc. SPIE 7483, 36.

Delaboudinière, J.-P., Artzner, G.E., Brunaud, J., Gabriel, A.H., Hochedez, J.F., Millier, F., Song, X.Y., Au, B., Dere, K.P., Howard, R.A., Kreplin, R., Michels, D.J., Moses, J.D., Defise, J.M., Jamar, C., Rochus, P., Chauvineau, J.P., Marioge, J.P., Catura, R.C., Lemen, J.R., Shing, L., Stern, R.A., Gurman, J.B., Neupert, W.M., Maucherat, A., Clette, F., Cugnon, P., van Dessel, E.L.: 1995, Solar Phys. 162, 291.

Dere, K.P., Landi, E., Mason, H.E., Monsignori Fossi, B.C., Young, P.R.: 1997, Astron. Astrophys. Suppl. 125, 149.

Dere, K.P., Landi, E., Young, P.R., Del Zanna, G., Landini, M., Mason, H.E.: 2009, Astron. Astrophys. 498, 915.

Freeland, S.L., Bentley, R.D.: 2000, Encyc. Astron. Astrophys., IOP Publishing, Bristol. doi:10.1888/ $0333750888 / 3390$.

Golub, L., DeLuca, E., Austin, G., Bookbinder, J., Caldwell, D., Cheimets, P., et al.: 2007, Solar Phys. 243, 63.

Gullikson, E.M., Mrowka, S., Kaufmann, B.B.: 2001, Proc. SPIE 4343, 363.

Handy, B.N., Acton, L.W., Kankelborg, C.C., Wolfson, C.J., Akin, D.J., Bruner, M.E., Caravalho, R., Catura, R.C., Chevalier, R., Duncan, D.W., Edwards, C.G., Feinstein, C.N., Freeland, S.L., Friedlaender, F.M., Hoffmann, C.H., Hurlburt, N.E., Jurcevich, B.K., Katz, N.L., Kelly, G.A., Lemen, J.R., Levay, M., Lindgren, R.W., Mathur, D.P., Meyer, S.B., Morrison, S.J., Morrison, M.D., Nightingale, R.W., Pope, T.P., Rehse, R.A., Schrijver, C.J., Shine, R.A., Shing, L., Strong, K.T., Tarbell, T.D., Title, A.M., Torgerson, D.D., Golub, L., Bookbinder, J.A., Caldwell, D., Cheimets, P.N., Davis, W.N., Deluca, E.E., McMullen, R.A., Warren, H.P., Amato, D., Fisher, R., Maldonado, H., Parkinson, C.: 1999, Solar Phys. 187, 229.

Howard, R.A., Moses, J.D., Vourlidas, A., Newmark, J.S., Socker, D.G., Plunkett, S.P., Korendyke, C.M., Cook, J.W., Hurley, A., Davila, J.M., Thompson, W.T., St Cyr, O.C., Mentzell, E., Mehalick, K., Lemen, J.R., Wuelser, J.P., Duncan, D.W., Tarbell, T.D., Wolfson, C.J., Moore, A., Harrison, R.A., Waltham, N.R., Lang, J., Davis, C.J., Eyles, C.J., Mapson-Menard, H., Simnett, G.M., Halain, J.P., Defise, J.M., Mazy, E., Rochus, P., Mercier, R., Ravet, M.F., Delmotte, F., Auchère, F., Delaboudinière, J.P., Bothmer, V., Deutsch, W., Wang, D., Rich, N., Cooper, S., Stephens, V., Maahs, G., Baugh, R., McMullin, D., Carter, T.: 2008, Space Sci. Rev. 136, 67.

Hurlburt, N., Cheung, M., Schrijver, C., Chang, L., Freeland, S., Green, S., Heck, C., Jaffey, A., Kobashi, A., Schiff, D., Serafin, J., Seguin, R., Slater, G., Somani, A., Timmons, R.: 2011, Solar Phys., doi:10.1007/ s11207-010-9624-2.

Lin, A.C., Nightingale, R.W., Tarbell, T.D.: 2001, Solar Phys. 198, 385.

Park, S.K., Schowengerdt, R.A.: 1983, Comput. Vis. Graph. Image Process. 23, 258.

Podgorski, W.A., Cheimets, P.N., Boerner, P., Glenn, P.: 2009, Proc. SPIE 7438, 13.

Schou, J., Scherrer, P.H., Bush, R.I., Wachter, R., Couvidat, S., Rabello-Soares, M.C., et al.: 2011, Solar Phys., in preparation.

Soufli, R., Windt, D.L., Robinson, J.C., Baker, S.L., Spiller, E.A., Dollar, F.J., Aquila, A.L., Gullikson, E.M., Kjornrattanawanich, B., Seely, J.F., Golub, L.: 2005, Proc. SPIE 5901, 59010M.

Soufli, R., Baker, S.L., Windt, D.L., Gullikson, E.M., Robinson, J.C., Podgorski, W.A., Golub, L.: 2007, Appl. Opt. 46, 3156.

Thompson, W.T.: 2006, Astron. Astrophys. 449, 791.

Thompson, W.T., Brekke, P.: 2000, Solar Phys. 195, 45.

Underwood, J.H., Gullikson, E.M., Koike, M., Batson, P.J.: 1997, Proc. SPIE 3113, 214.

Waltham, N., Beardsley, S., Clapp, M., Lang, J., Jerram, P., Pool, P., Auker, G., Morris, D., Duncan, D.: 2011, In: Internat. Conf. on Space Optics, in press.

Woods, T.N., Eparvier, F.G., Hock, R., Jones, A.R., Woodraska, D., Judge, D., Didkovsky, L., Lean, J., Mariska, J., Warren, H., McMullin, D., Chamberlin, P., Berthiaume, G., Bailey, S., Fuller-Rowell, T., Sojka, J., Tobiska, W.K., Viereck, R.: 2011, Solar Phys., doi:10.1007/s11207-009-9487-6. 